\title{
Finite Element Solutions for the Space Fractional Diffusion Equation with a Nonlinear Source Term
}

\author{
Y. J. Choi and S. K. Chung \\ Department of Mathematics Education, Seoul National University, Seoul 151-748, Republic of Korea \\ Correspondence should be addressed to S. K. Chung, chung@snu.ac.kr
}

Received 26 April 2012; Revised 6 July 2012; Accepted 17 July 2012

Academic Editor: Bashir Ahmad

Copyright (c) 2012 Y. J. Choi and S. K. Chung. This is an open access article distributed under the Creative Commons Attribution License, which permits unrestricted use, distribution, and reproduction in any medium, provided the original work is properly cited.

We consider finite element Galerkin solutions for the space fractional diffusion equation with a nonlinear source term. Existence, stability, and order of convergence of approximate solutions for the backward Euler fully discrete scheme have been discussed as well as for the semidiscrete scheme. The analytical convergent orders are obtained as $O(k+h \tilde{r})$, where $\tilde{\gamma}$ is a constant depending on the order of fractional derivative. Numerical computations are presented, which confirm the theoretical results when the equation has a linear source term. When the equation has a nonlinear source term, numerical results show that the diffusivity depends on the order of fractional derivative as we expect.

\section{Introduction}

Fractional calculus is an old mathematical topic but it has not been attracted enough for almost three hundred years. However, it has been recently proven that fractional calculus is a significant tool in the modeling of many phenomena in various fields such as engineering, physics, porous media, economics, and biological sciences. One can see related references in [1-7].

In the classical diffusion model, it is assumed that particles are distributed in a normal bell-shaped pattern based on the Brownian motion. In general, the nature of diffusion is characterized by the mean squared displacement

$$
\left\langle(\Delta r)^{2}\right\rangle=2 d \kappa_{\mu} t^{\mu}
$$

where $d$ is the spatial dimension and $\kappa_{\mu}$ is the diffusion constant. The classical normal diffusion case arises when the exponent $\mu=1$. When $\mu \neq 1$, anomalous diffusions arise. 
The anomalous diffusion is classified as the process is subdiffusive (diffusive slowly) when $\mu<1$ or superdiffusive (diffusive fast) when $\mu>1$.

As mentioned before, in many real problems, it is more adequate to use anomalous diffusion described by fractional derivatives than the classical normal diffusion $[4,5,8-12]$. One typical model for anomalous diffusion is the fractional superdiffusion equation arising in chaotic and turbulent processes, where the usual second derivative in space is replaced by a fractional derivative of order $1<\mu<2$.

In this paper we discuss Galerkin approximate solutions for the space fractional diffusion equation with a nonlinear source term. The equation is described as

$$
\frac{\partial u(x, t)}{\partial t}=\kappa_{\mu} \nabla^{\mu} u(x, t)+f(x, t, u)
$$

with an initial condition

$$
u(x, 0)=u_{0}, \quad x \in \Omega \subset \mathbf{R}
$$

and boundary conditions

$$
u(x, t)=0, \quad x \in \partial \Omega, 0 \leq t \leq T,
$$

where $\kappa_{\mu}$ denotes the anomalous diffusion coefficient and $\partial \Omega$ is the boundary of the domain $\Omega$. And the differential operator $\nabla^{\mu}$ is

$$
\nabla^{\mu}=\frac{1}{2}{ }_{a} D_{x}^{\mu}+\frac{1}{2}{ }_{x} D_{b^{\prime}}^{\mu}
$$

where ${ }_{a} D_{x}^{\mu}$ and ${ }_{x} D_{b}^{\mu}$ are called the left and the right Riemann-Liouville space fractional derivatives of order $\mu$, respectively, defined by

$$
\begin{gathered}
\mathbf{D}^{\mu} u:={ }_{a} D_{x}^{\mu} u(x)=D_{a}^{n} D_{x}^{\mu-n} u(x)=\frac{1}{\Gamma(n-\mu)} \frac{d^{n}}{d x^{n}} \int_{a}^{x}(x-\xi)^{n-\mu-1} u(\xi) d \xi, \\
\mathbf{D}^{\mu *} u:={ }_{x} D_{b}^{\mu} u(x)=(-D)^{n}{ }_{x} D_{b}^{\mu-n} u(x)=\frac{(-1)^{n}}{\Gamma(n-\mu)} \frac{d^{n}}{d x^{n}} \int_{x}^{b}(\xi-x)^{n-\mu-1} u(\xi) d \xi .
\end{gathered}
$$

Here $n$ is the smallest integer such that $n-1 \leq \mu<n$.

Throughout this paper, we will assume that the nonlinear source term $f(x, t, u)$ is locally Lipschitz continuous with constants $C_{l}$ and $C_{f}$ such that

$$
\begin{gathered}
\|f(u)-f(v)\|_{L^{2}(\Omega)} \leq C_{l}\|u-v\|_{L^{2}(\Omega)}, \\
\|f(u)\|_{L^{2}(\Omega)} \leq C_{f}\|u\|_{L^{2}(\Omega)}
\end{gathered}
$$

for $u, v \in\left\{w \in H_{0}^{\mu / 2}(\Omega) \mid\|w\|_{L^{2}(\Omega)} \leq l\right\}$. 
Baeumer et al. $[8,13]$ have proved existence and uniqueness of a strong solution for (1.2) using the semigroup theory when $f(x, t, u)$ is globally Lipschitz continuous. Furthermore, when $f(x, t, u)$ is locally Lipschitz continuous, existence of a unique strong solution has also been shown by introducing the cut-off function.

Finite difference methods have been studied in [14-16] for linear space fractional diffusion problems. They used the right-shifted Grüwald-Letnikov approximate for the fractional derivative since the standard Grüwald-Letnikov approximate gives the unconditional instability even for the implicit method. Using the right-shifted Grüwald-Letnikov approximation, the method of lines has been applied in [12] for numerical approximate solutions.

For the space fractional diffusion problems with a nonlinear source term, Lynch et al. [17] used the so-called L2 and L2C methods in [6] and compared computational accuracy of them. Baeumer et al. [8] give existence of the solution and computational results using finite difference methods. Choi et al. [18] have shown existence and stability of numerical solutions of an implicit finite difference equation obtained by using the right-shifted Grüwald-Letnikov approximation. For the time fractional diffusion equations, explicit and implicit finite difference methods have been used in [11, 19-23].

Compared to finite difference methods on the fractional diffusion equation, finite element methods have been rarely discussed. Ervin and Roop [24] have considered finite element analysis for stationary linear advection dispersion equations, and Roop [25] has studied finite element analysis for nonstationary linear advection dispersion equations. The finite element numerical approximations have been discussed for the time and space fractional Fokker-Planck equation in Deng [9] and for the space general fractional diffusion equations with a nonlocal quadratic nonlinearity but a linear source term in Ervin et al. [26].

As far as we know, finite element methods have not been considered for the space fractional diffusion equation with nonlinear source terms. In this paper, we will discuss finite element solutions for the problem (1.2)-(1.4) under the assumption of existence of a sufficiently regular solution $u$ of the equation. Finite element numerical analysis of the semidiscrete and fully discrete methods for (1.2)-(1.4) will be considered using the backward Euler method in time and Galerkin finite element method in space as well as the semidiscrete method. We will discuss existence, uniqueness, and stability of the numerical solutions for the problem (1.2)-(1.4). Also, $L^{2}$-error estimate will be considered for the problem (1.2)-(1.4).

The outline of the paper is as follows. We introduce some properties of the space fractional derivatives in Section 2, which will be used in later discussion. In Section 3, the semidiscrete variational formulation for (1.2) based on Galerkin method is given. Existence, stability and $L^{2}$-error estimate of the semidiscrete solution are analyzed. In Section 4 , existence and unconditional stability of approximate solutions for the fully discrete backward Euler method are shown following the idea of the semidiscrete method. Further, $L^{2}$-error estimates are obtained, whose convergence is of $O(k+h \tilde{r})$, where $\tilde{\gamma}=\mu$ if $\mu \neq 3 / 2$ and $\tilde{\gamma}=$ $\mu-\epsilon, 0<\epsilon<1 / 2$, if $\mu=3 / 2$. Finally, numerical examples are given in order to see the theoretical convergence order discussed in Section 5. We will see that numerical solutions of fractional diffusion equations diffuse more slowly than that of the classical diffusion problem and diffusivity depends on the order of fractional derivatives.

\section{The Variational Form}

In this section we will consider the variational form of problem (1.2)-(1.4) and show existence and stability of the weak solution. We first recall some basic properties of Riemann-Liouville fractional calculus $[9,24]$. 
For any given positive number $\mu>0$, define the seminorm

$$
|u|_{J_{L}^{\mu}(\mathbf{R})}=\left\|\mathbf{D}^{\mu} u\right\|_{L^{2}(\mathbf{R})}
$$

and the norm

$$
\|u\|_{J_{L}^{\mu}(\mathbf{R})}=\left(\|u\|_{L^{2}(\mathbf{R})}^{2}+|u|_{J_{L}^{\mu}(\mathbf{R})}^{2}\right)^{1 / 2}
$$

where the left fractional derivative space $J_{L}^{\mu}(\mathbf{R})$ denotes the closure of $C_{0}^{\infty}(\mathbf{R})$ with respect to the norm $\|\cdot\|_{J_{L}^{\mu}(\mathbf{R})}$.

Similarly, we may define the right fractional derivative space $J_{R}^{\mu}(\mathbf{R})$ as the closure of $C_{0}^{\infty}(\mathbf{R})$ with respect to the norm $\|\cdot\|_{J_{R}^{\mu}(\mathbf{R})}$, where

$$
\|u\|_{J_{R}^{\mu}(\mathbf{R})}=\left(\|u\|_{L^{2}(\mathbf{R})}^{2}+|u|_{J_{R}^{\mu}(\mathbf{R})}^{2}\right)^{1 / 2}
$$

and the seminorm

$$
|u|_{J_{R}^{\mu}(\mathbf{R})}=\left\|\mathbf{D}^{\mu *} u\right\|_{L^{2}(\mathbf{R})} .
$$

Furthermore, with the help of Fourier transform we define a seminorm

$$
|u|_{H^{\mu}(\mathbf{R})}=\left\||\omega|^{\mu} \widehat{u}\right\|_{L^{2}(\mathbf{R})}
$$

and the norm

$$
\|u\|_{H^{\mu}(\mathbf{R})}=\left(\|u\|_{L^{2}(\mathbf{R})}^{2}+|u|_{H^{\mu}(\mathbf{R})}^{2}\right)^{1 / 2}
$$

Here $H^{\mu}(\mathbf{R})$ denotes the closure of $C_{0}^{\infty}(\mathbf{R})$ with respect to $\|\cdot\|_{H^{\mu}(\mathbf{R})}$. It is known in [24] that the spaces $J_{L}^{\mu}(\mathbf{R}), J_{R}^{\mu}(\mathbf{R})$, and $H^{\mu}(\mathbf{R})$ are all equal with equivalent seminorms and norms. Analogously, when the domain $\Omega$ is a bounded interval, the spaces $J_{L, 0}^{\mu}(\Omega), J_{R, 0}^{\mu}(\Omega)$, and $H_{0}^{\mu}(\Omega)$ are equal with equivalent seminorms and norms [24, 27].

The following lemma on the Riemann-Liouville fractional integral operators will be used in our analysis, which can be proved by using the property of Fourier transform [24].

Lemma 2.1. For a given $\mu>0$ and a real valued function $u$

$$
\left(\mathbf{D}^{\mu} u, \mathbf{D}^{\mu *} u\right)=\cos (\pi \mu)\left\|\mathbf{D}^{\mu} u\right\|_{L^{2}(\mathbf{R})}^{2} .
$$

Remark 2.2. It follows from (2.7) that we may use the following norm:

$$
\|u\|_{H_{0}^{\mu / 2}(\mathbf{R})}^{2}=\|u\|_{L_{2}(\mathbf{R})}^{2}+\kappa_{\mu}\left|\cos \left(\pi \cdot \frac{\mu}{2}\right)\right||u|_{H_{0}^{\mu / 2}(\mathbf{R})}^{2}
$$

instead of the norm $\|u\|_{H^{\mu}(\mathbf{R})}$. 
For the seminorm on $H_{0}^{\mu}(\Omega)$ with $\Omega=(a, b)$, the following fractional PoincaréFriedrich's inequality holds. For the proof, we refer to $[9,24]$.

Lemma 2.3. For $u \in H_{0}^{\mu}(\Omega)$, there is a positive constant $C$ such that

$$
\|u\|_{L^{2}(\Omega)} \leq C|u|_{H_{0}^{\mu}(\Omega)}
$$

and for $0<s<\mu, s \neq n-1 / 2, n-1 \leq \mu<n, n \in \mathbb{N}$,

$$
|u|_{H_{0}^{s}(\Omega)} \leq C|u|_{H_{0}^{\mu}(\Omega)}
$$

Hereafter, a positive number $C$ will denote a generic constant. Also the semigroup property and the adjoint property hold for the Riemann-Liouville fractional integral operators $[9,24]$ : for all $\mu, v>0$, if $u \in L^{p}(\Omega), p \geq 1$, then

$$
\begin{aligned}
& { }_{a} D_{x}^{-\mu}{ }_{a} D_{x}^{-v} u(x)={ }_{a} D_{x}^{-\mu-v} u(x), \quad \forall x \in \Omega, \\
& { }_{x} D_{b}^{-\mu}{ }_{x} D_{b}^{-v} u(x)={ }_{x} D_{b}^{-\mu-v} u(x), \quad \forall x \in \Omega,
\end{aligned}
$$

and specially

$$
\left({ }_{a} D_{x}^{-\mu} u, v\right)_{L^{2}(\Omega)}=\left(u,{ }_{x} D_{b}^{-\mu} v\right)_{L^{2}(\Omega)^{\prime}} \quad \forall u, v \in L^{2}(\Omega) .
$$

In the rest of this section, we will consider a weak problem for (1.2)-(1.4) with $1<\mu<$ 2: find a function $u \in H_{0}^{\mu / 2}(\Omega)$ such that

$$
\left(u_{t}, v\right)=\left(\kappa_{\mu} \nabla^{\mu} u, v\right)+(f(u), v), \quad \forall v \in H_{0}^{\mu / 2}(\Omega) .
$$

Since there is a weak solution of (2.13) when $f$ is locally Lipschitz continuous as in $[8,13]$, we here only discuss the stability of the weak solution, to show that we need the following lemma.

Lemma 2.4. For all $v \in H_{0}^{\mu / 2}(\Omega)$, the following inequality holds:

$$
-\left(\kappa_{\mu} \nabla^{\mu} v, v\right) \geq \kappa_{\mu}\left|\cos \left(\pi \cdot \frac{\mu}{2}\right)\right||v|_{H_{0}^{\mu / 2}(\Omega)}^{2} .
$$


Proof. Following the ideas in $[9,26]$, we obtain the following inequality by using the properties (2.11)-(2.12) and Lemmas 2.1-2.3:

$$
\begin{aligned}
-\left(\kappa_{\mu} \nabla^{\mu} v, v\right)= & -\frac{\kappa_{\mu}}{2}\left\{\left({ }_{a} D_{x}^{\mu} v, v\right)+\left({ }_{x} D_{b}^{\mu} v, v\right)\right\} \\
= & -\frac{\kappa_{\mu}}{2}\left\{\int_{a}^{b}\left(D^{2}{ }_{a} D_{x}^{-(2-\mu)} v\right) v d x+\int_{a}^{b}\left((-D)^{2}{ }_{x} D_{b}^{-(2-\mu)} v\right) v d x\right\} \\
= & \frac{\kappa_{\mu}}{2}\left\{\int_{a}^{b}\left(D_{a} D_{x}^{-(2-\mu)} v\right) D v d x+\int_{a}^{b}\left(D_{x} D_{b}^{-(2-\mu)} v\right) D v d x\right\} \\
= & \frac{\kappa_{\mu}}{2}\left\{\int_{a}^{b}\left({ }_{a} D_{x}^{-(2-\mu)} D v\right) D v d x+\int_{a}^{b}\left({ }_{x} D_{b}^{-(2-\mu)} D v\right) D v d x\right\} \\
= & \frac{\kappa_{\mu}}{2}\left\{\int_{a}^{b}\left({ }_{a} D_{x}^{-(2-\mu) / 2}{ }_{a} D_{x}^{-(2-\mu) / 2} D v\right) D v d x\right. \\
& \left.\quad+\int_{a}^{b}\left({ }_{x} D_{b}^{-((2-\mu) / 2)}{ }_{x} D_{b}^{-(2-\mu) / 2} D v\right) D v d x\right\} \\
= & \frac{\kappa_{\mu}}{2}\left\{\int_{a}^{b}\left({ }_{a} D_{x}^{-(2-\mu) / 2} D v\right)\left({ }_{x} D_{b}^{-(2-\mu) / 2} D v\right) d x\right. \\
& \left.\quad+\int_{a}^{b}\left({ }_{x} D_{b}^{-(2-\mu) / 2} D v\right)\left({ }_{a} D_{x}^{-(2-\mu) / 2} D v\right) d x\right\} \\
= & -\kappa_{\mu}\left(\mathbf{D}^{\mu / 2} v, \mathbf{D}^{(\mu / 2) *} v\right) \\
= & -\left.\kappa_{\mu} \cos \left(\pi \cdot \frac{\mu}{2}\right)\left\|\mathbf{D}^{\mu / 2} v\right\|_{L^{2}(\Omega)}^{2}\left(\pi \cdot \frac{\mu}{2}\right)|| v\right|_{H_{0}^{\mu / 2}(\Omega)} \cdot \\
& \\
& \\
&
\end{aligned}
$$

This completes the proof.

We consider the stability of a weak solution $u$ for (2.13).

Theorem 2.5. Let $u$ be a solution of (2.13). Then there is a constant $C$ such that

$$
\|u(t)\|_{L^{2}(\Omega)} \leq C\|u(0)\|_{L^{2}(\Omega)} .
$$

Proof. Taking $v=u(t)$ in (2.13), we obtain

$$
\left(u_{t}, u\right)-\left(\kappa_{\mu} \nabla^{\mu} u, u\right)=(f(u), u) .
$$


Since the second term on the left hand side is nonnegative from Lemma 2.4, we have

$$
\begin{aligned}
\frac{1}{2} \frac{d}{d t}\|u\|_{L^{2}(\Omega)}^{2} & \leq \frac{1}{2} \frac{d}{d t}\|u\|_{L^{2}(\Omega)}^{2}+\kappa_{\mu}\left|\cos \left(\pi \cdot \frac{\mu}{2}\right)\right||u|_{H_{0}^{\mu / 2}(\Omega)}^{2} \\
& \leq\|f(u)\|_{L^{2}(\Omega)}\|u\|_{L^{2}(\Omega)} \\
& \leq C_{f}\|u\|_{L^{2}(\Omega)}^{2}
\end{aligned}
$$

Integrating both sides with respect to $t$, we obtain

$$
\|u(t)\|_{L^{2}(\Omega)}^{2} \leq\|u(0)\|_{L^{2}(\Omega)}^{2}+C \int_{0}^{t}\|u(s)\|_{L^{2}(\Omega)}^{2} d s
$$

An application of Gronwall's inequality gives that there is a constant $C$ such that

$$
\|u(t)\|_{L^{2}(\Omega)}^{2} \leq C\|u(0)\|_{L^{2}(\Omega)}^{2} .
$$

This completes the proof.

\section{The Semidiscrete Variational Form}

In this section, we will analyze the stability and error estimates of Galerkin finite element solutions for the semidiscrete variational formulation for (1.2).

Let $S_{h}$ be a partition of $\Omega$ with a grid parameter $h$ such that $\bar{\Omega}=\left\{\cup K \mid K \in S_{h}\right\}$ and $h=\max _{K \in S_{h}} h_{K}$, where $h_{K}$ is the width of the subinterval $K$. Associated with the partition $S_{h}$, we may define a finite-dimensional subspace $V_{h} \subset H_{0}^{\mu / 2}(\Omega)$ with a basis $\left\{\varphi_{i}\right\}_{i=1}^{N}$ of piecewise polynomials. Then the semidiscrete variational problem is to find $u_{h} \in V_{h}$ such that

$$
\begin{gathered}
\left(u_{h, t}, v\right)=\left(\kappa_{\mu} \nabla^{\mu} u_{h}, v\right)+\left(f\left(u_{h}\right), v\right), \quad \forall v \in V_{h}, \\
u_{h}(x, 0)=u_{0}, \\
u_{h}(a, t)=u_{h}(b, t)=0 .
\end{gathered}
$$

Since $u_{h}$ can be represented as

$$
u_{h}(x, t)=\sum_{i=1}^{N} \alpha_{i}(t) \varphi_{i}(x)
$$

we may rewrite (3.1) in a matrix form:

$$
\mathbf{A} \dot{\mathbf{u}}(t)+\mathbf{B} \mathbf{u}=\mathbf{F}(\mathbf{u}),
$$


where $N \times N$ matrices $\mathbf{A}$ and $\mathbf{B}$ and vectors $\mathbf{u}$ and $\mathbf{F}$ are

$$
\begin{gathered}
\mathbf{A}=\left(a_{i j}\right), \quad a_{i j}=\left(\varphi_{i}, \varphi_{j}\right), \\
\mathbf{B}=\left(b_{i j}\right), \quad b_{i j}=-\frac{\mathcal{K}_{\mu}}{2}\left[\left(\mathbf{D}^{\mu / 2} \varphi_{i}, \mathbf{D}^{(\mu / 2) *} \varphi_{j}\right)+\left(\mathbf{D}^{\mu / 2} \varphi_{j}, \mathbf{D}^{(\mu / 2) *} \varphi_{i}\right)\right] \\
\mathbf{F}(\mathbf{u})=\left(F_{j}\right), \quad F_{j}=\left(f\left(\sum_{l=1}^{N} \alpha_{l} \varphi_{l}\right), \varphi_{j}\right), \\
\mathbf{u}=\left(\alpha_{1}(t), \alpha_{2}(t), \ldots, \alpha_{N}(t)\right)^{T} .
\end{gathered}
$$

It follows from $\sum_{i, j=1}^{N} \alpha_{i} \alpha_{j}\left(\varphi_{i}, \varphi_{j}\right)=\left(\sum_{i=1}^{N} \alpha_{i} \varphi_{i}, \sum_{j=1}^{N} \alpha_{j} \varphi_{j}\right) \geq 0$ and Lemma 2.4 that matrices $\mathbf{A}$ and $\mathbf{B}$ are nonnegative definite and nonsingular. Thus this system (3.5) of ordinary differential equations has a unique solution since $f$ is locally Lipschitz continuous.

The stability for the semidiscrete variational problem (3.1) can be obtained by following the proof of Theorem 2.5, which is

$$
\left\|u_{h}\right\|_{L^{2}(\Omega)} \leq C\left\|u_{0}\right\|_{L^{2}(\Omega)}
$$

Now we will consider estimates of error between the weak solution of (2.13) and the one of semidiscrete form (3.1). The finite dimensional subspace $V_{h} \subset H_{0}^{\mu / 2}(\Omega)$ is chosen so that the interpolation $I^{h} u$ of $u$ satisfies an approximation property [9, 28]: for $u \in H^{\gamma}(\Omega)$, $0<\gamma \leq n$, and $0 \leq s \leq \gamma$, there exists a constant $C$ depending only on $\Omega$ such that

$$
\left\|u-I^{h} u\right\|_{H^{s}(\Omega)} \leq C h^{r-s}\|u\|_{H^{r}(\Omega)} .
$$

Since the norm $\|\cdot\|_{H^{s}(\Omega)}$ is equivalent to the seminorm $|\cdot|_{H^{s}(\Omega)}$, we may replace (3.8) by the relation

$$
\left\|u-I^{h} u\right\|_{H^{s}(\Omega)} \leq C h^{\gamma-s}|u|_{H^{r}(\Omega)}
$$

Further we need an adjoint problem to find $w \in H^{\mu}(\Omega) \cap H_{0}^{\mu / 2}(\Omega)$ satisfying

$$
\begin{gathered}
-\kappa_{\mu} \nabla^{\mu} w=g, \quad \text { in } \Omega, \\
w=0, \quad \text { on } \partial \Omega .
\end{gathered}
$$

Bai and Lü [29] have proved existence of a solution to the problem (3.10). We assume as in Ervin and Roop [24] that the solution $w$ satisfies the regularity

$$
\begin{gathered}
\|w\|_{H^{\mu}(\Omega)} \leq C\|g\|_{L^{2}(\Omega)}, \quad \mu \neq \frac{3}{2}, \\
\|w\|_{H^{\mu-\epsilon}(\Omega)} \leq C\|g\|_{L^{2}(\Omega)^{\prime}}, \quad \mu=\frac{3}{2}, \quad 0<\epsilon<\frac{1}{2} .
\end{gathered}
$$


Let $\tilde{u}_{h}=P_{h} u$ be the elliptic projection $P_{h}: H_{0}^{\mu / 2}(\Omega) \rightarrow V_{h}$ of the exact solution $u$, which is defined by

$$
-\kappa_{\mu}\left(\nabla^{\mu}\left(u-\tilde{u}_{h}\right), v\right)=0, \quad \forall v \in V_{h}
$$

Let $\theta=u_{h}-\tilde{u}_{h}$ and $\rho=\tilde{u}_{h}-u$. Then the error is expressed as

$$
e_{h}=u_{h}-u=\left(u_{h}-\tilde{u}_{h}\right)+\left(\tilde{u}_{h}-u\right)=\theta+\rho .
$$

First, we consider the following estimates on $\rho$.

Lemma 3.1. Let $\tilde{u}_{h}$ be a solution of (3.13) and let $u \in H^{\mu}(\Omega) \cap H_{0}^{\mu / 2}(\Omega)$ be the solution of (2.13). Let $\rho(t)=\tilde{u}_{h}(t)-u(t)$. Then there is a constant $C$ such that

$$
\begin{aligned}
\|\rho(t)\|_{L^{2}(\Omega)} & \leq C h^{\tilde{r}}\|u(t)\|_{H^{r}(\Omega)}, \\
\left\|\rho_{t}(t)\right\|_{L^{2}(\Omega)} & \leq C h^{\tilde{r}}\|u(t)\|_{H^{r}(\Omega)},
\end{aligned}
$$

where $\tilde{\gamma}=\mu$ if $\mu \neq 3 / 2$ and $\tilde{\gamma}=\mu-\epsilon, 0<\epsilon<1 / 2$ if $\mu=3 / 2$.

Proof. It follows from the fractional Poincare-Friedrich's inequality and the adjoint property (2.12) that for $\psi, \chi \in V_{h} \subset H_{0}^{\mu / 2}(\Omega)$

$$
\begin{aligned}
\left(\mathbf{D}^{\mu} \psi, X\right) & =\int_{a}^{b}\left(\mathbf{D}^{\mu / 2} \psi\right) \mathbf{D}^{(\mu / 2) *} X d x \\
& \leq|\psi|_{J_{L, 0}^{\mu / 2}(\Omega)}|X|_{J_{R, 0}^{\mu / 2}(\Omega)} \\
& \leq C\|\psi\|_{H_{0}^{\mu / 2}(\Omega)}\|X\|_{H_{0}^{\mu / 2}(\Omega)} .
\end{aligned}
$$

Similarly we obtain

$$
\left(\mathbf{D}^{\mu *} \psi, X\right)=\int_{a}^{b}\left(\mathbf{D}^{(\mu / 2) *} \psi\right) \mathbf{D}^{\mu / 2} \chi d x \leq C\|\psi\|_{H_{0}^{\mu / 2}(\Omega)}\|X\|_{H_{0}^{\mu / 2}(\Omega)} .
$$

It follows from Lemma 2.4 that for $v \in V_{h}$

$$
\begin{aligned}
\kappa_{\mu}\left|\cos \left(\pi \cdot \frac{\mu}{2}\right)\right|\left|u-\tilde{u}_{h}\right|_{H_{0}^{\mu / 2}(\Omega)}^{2} & \leq-\kappa_{\mu}\left(\nabla^{\mu}\left(u-\tilde{u}_{h}\right), u-\tilde{u}_{h}\right) \\
& \leq-\kappa_{\mu}\left(\nabla^{\mu}\left(u-\tilde{u}_{h}\right), u-v\right)-\kappa_{\mu}\left(\nabla^{\mu}\left(u-\tilde{u}_{h}\right), v-\tilde{u}_{h}\right) \\
& \leq C\left\|u-\tilde{u}_{h}\right\|_{H_{0}^{\mu / 2}(\Omega)}\|u-v\|_{H_{0}^{\mu / 2}(\Omega)} .
\end{aligned}
$$


Using the equivalence of seminorms and norms, we obtain

$$
\left\|u-\tilde{u}_{h}\right\|_{H_{0}^{\mu / 2}(\Omega)} \leq C \inf _{v \in V_{h}}\|u-v\|_{H_{0}^{\mu / 2}(\Omega)} \leq C\left\|u-I^{h} u\right\|_{H_{0}^{\mu / 2}(\Omega)} .
$$

In case of $\mu \neq 3 / 2$ and $v \in V_{h}$, by taking $g=\rho$ in (3.10) and using (3.13), (3.16)-(3.17) and the adjoint property (2.12), we have

$$
\begin{aligned}
(\rho, \rho) & =-\kappa_{\mu}\left(\nabla^{\mu} w, \rho\right) \\
& =-\kappa_{\mu}\left(\nabla^{\mu}(w-v), \rho\right)-\kappa_{\mu}\left(\nabla^{\mu} \rho, v\right) \\
& =-\kappa_{\mu}\left(\nabla^{\mu}(w-v), \rho\right) \\
& \leq C\|w-v\|_{H_{0}^{\mu / 2}(\Omega)}\|\rho\|_{H_{0}^{\mu / 2}(\Omega)} .
\end{aligned}
$$

Taking $v=I^{h} w$ in the previously mentioned inequalities, we have

$$
\begin{aligned}
\|\rho\|_{L^{2}(\Omega)}^{2} & \leq C\left\|w-I^{h} w\right\|_{H_{0}^{\mu / 2}(\Omega)}\|\rho\|_{H_{0}^{\mu / 2}(\Omega)} \\
& \leq C h^{\mu / 2}\|w\|_{H^{\mu}(\Omega)}\left\|u-I^{h} u\right\|_{H_{0}^{\mu / 2}(\Omega)} \\
& \leq C h^{\mu / 2}\|\rho\|_{L^{2}(\Omega)} h^{\mu / 2}\|u\|_{H^{\mu}(\Omega)} .
\end{aligned}
$$

Thus we obtain

$$
\|\rho\|_{L^{2}(\Omega)} \leq C h^{\mu}\|u\|_{H^{\mu}(\Omega)}
$$

We now differentiate (3.13). Then we obtain $-\kappa_{\mu}\left(\nabla^{\mu} \rho_{t}, v\right)=0$ for all $v \in V_{h}$. Using the previous duality arguments again, we have

$$
\left\|\rho_{t}\right\|_{L^{2}(\Omega)} \leq C h^{\mu}\|u\|_{H^{\mu}(\Omega)} .
$$

In case of $\mu=3 / 2$, we can similarly prove (3.15) by applying the assumption (3.12). This completes the proof.

We now consider the estimates on $\theta$.

Lemma 3.2. Let $u_{h}$ and $\tilde{u}_{h}$ be the solutions of (3.1)-(3.3) and (3.13), respectively. Let $\theta(t)=u_{h}(t)-$ $\tilde{u}_{h}(t)$. Then there is a constant $C$ such that

$$
\|\theta(t)\|_{L^{2}(\Omega)} \leq C h^{\tilde{r}},
$$

where $\tilde{\gamma}=\mu$ if $\mu \neq 3 / 2$ and $\tilde{\gamma}=\mu-\epsilon, 0<\epsilon<1 / 2$ if $\mu=3 / 2$. 
Proof. It follows from (3.1) and (3.13) that for $v \in V_{h}$,

$$
\left(\theta_{t}, v\right)-\kappa_{\mu}\left(\nabla^{\mu} \theta, v\right)=\left(f\left(u_{h}\right)-f(u), v\right)-\left(\rho_{t}, v\right)
$$

Replacing $v=\theta$ in (3.25), we obtain

$$
\frac{1}{2} \frac{d}{d t}\|\theta\|_{L^{2}(\Omega)}^{2} \leq C_{l}\left\|u_{h}-u\right\|_{L^{2}(\Omega)}\|\theta\|_{L^{2}(\Omega)}+\left\|\rho_{t}\right\|_{L^{2}(\Omega)}\|\theta\|_{L^{2}(\Omega)} .
$$

Using Young's inequality

$$
\begin{aligned}
\frac{d}{d t}\|\theta\|_{L^{2}(\Omega)}^{2} & \leq C\left(\left\|u_{h}-\tilde{u}_{h}\right\|_{L^{2}(\Omega)}+\left\|\tilde{u}_{h}-u\right\|_{L^{2}(\Omega)}\right)\|\theta\|_{L^{2}(\Omega)}+\left\|\rho_{t}\right\|_{L^{2}(\Omega)}\|\theta\|_{L^{2}(\Omega)} \\
& \leq C\left(\|\theta\|_{L^{2}(\Omega)}+\|\rho\|_{L^{2}(\Omega)}+\left\|\rho_{t}\right\|_{L^{2}(\Omega)}\right)\|\theta\|_{L^{2}(\Omega)} \\
& \leq C_{1}\|\theta\|_{L^{2}(\Omega)}^{2}+C_{2}\|\rho\|_{L^{2}(\Omega)}^{2}+C_{3}\left\|\rho_{t}\right\|_{L^{2}(\Omega)}^{2} .
\end{aligned}
$$

Integration on time $t$ gives

$$
\|\theta(t)\|_{L^{2}(\Omega)}^{2} \leq\|\theta(0)\|_{L^{2}(\Omega)}^{2}+C \int_{0}^{t}\|\theta\|_{L^{2}(\Omega)}^{2} d s+C \int_{0}^{t}\left(\|\rho\|_{L^{2}(\Omega)}^{2}+\left\|\rho_{t}\right\|_{L^{2}(\Omega)}^{2}\right) d s .
$$

Applying Gronwall's inequality, we obtain

$$
\|\theta(t)\|_{L^{2}(\Omega)}^{2} \leq C_{1}\|\theta(0)\|_{L^{2}(\Omega)}^{2}+C_{2} \int_{0}^{t}\left(\|\rho\|_{L^{2}(\Omega)}^{2}+\left\|\rho_{t}\right\|_{L^{2}(\Omega)}^{2}\right) d s
$$

Since

$$
\begin{aligned}
\|\theta(0)\|_{L^{2}(\Omega)} & \leq\left\|u_{h}(0)-u(0)\right\|_{L^{2}(\Omega)}+\left\|\tilde{u}_{h}(0)-u(0)\right\|_{L^{2}(\Omega)} \\
& \leq C h^{\tilde{r}}\left\|u_{0}\right\|_{H^{r}(\Omega)},
\end{aligned}
$$

we obtain the desired inequality

$$
\|\theta(t)\|_{L^{2}(\Omega)} \leq C h^{\tilde{r}},
$$

where $\tilde{\gamma}=\mu$ if $\mu \neq 3 / 2$ and $\tilde{\gamma}=\mu-\epsilon, 0<\epsilon<1 / 2$, if $\mu=3 / 2$. 
Combining Lemmas 3.1 and 3.2, we obtain the following error estimates.

Theorem 3.3. Let $u_{h}$ and $u$ be the solutions of (3.1)-(3.3) and (1.2)-(1.4), respectively. Then there is a constant $C(u)$ such that

$$
\begin{gathered}
\left\|u(t)-u_{h}(t)\right\|_{L^{2}(\Omega)} \leq C(u) h^{\mu}, \quad \mu \neq \frac{3}{2}, \\
\left\|u(t)-u_{h}(t)\right\|_{L^{2}(\Omega)} \leq C(u) h^{\mu-\epsilon}, \quad \mu=\frac{3}{2}, \quad 0<\epsilon<\frac{1}{2} .
\end{gathered}
$$

\section{The Fully Discrete Variational Form}

In this section, we consider a fully discrete variational formulation of (1.2). Existence and uniqueness of numerical solutions for the fully discrete variational formulation are discussed. The corresponding error estimates are also analyzed.

For the temporal discretization let $k=T / M$ for a positive integer $M$ and $t_{m}=m k$. Let $u^{m}$ be the solution of the backward Euler method defined by

$$
\frac{u^{m+1}-u^{m}}{k}=\kappa_{\mu} \nabla^{\mu} u^{m+1}+f\left(u^{m+1}\right)
$$

with an initial condition

$$
u^{0}(x)=u_{0}, \quad x \in \Omega=(a, b)
$$

and boundary conditions

$$
u^{m+1}(a)=u^{m+1}(b)=0, \quad m=0,1, \ldots, M-1 .
$$

Then we get the fully discrete variational formulation of (1.2) to find $u^{m+1} \in H_{0}^{\mu / 2}(\Omega)$ such that for all $v \in H_{0}^{\mu / 2}(\Omega)$

$$
\left(u^{m+1}, v\right)-k\left(\kappa_{\mu} \nabla^{\mu} u^{m+1}, v\right)=\left(k f\left(u^{m+1}\right), v\right)+\left(u^{m}, v\right)
$$

Thus a finite Galerkin solution $u_{h}^{m+1} \in V_{h} \subset H_{0}^{\mu / 2}(\Omega)$ is a solution of the equation

$$
\left(u_{h}^{m+1}, v_{h}\right)-k \kappa_{\mu}\left(\nabla^{\mu} u_{h}^{m+1}, v_{h}\right)=k\left(f\left(u_{h}^{m+1}\right), v_{h}\right)+\left(u_{h}^{m}, v_{h}\right), \quad \forall v_{h} \in V_{h}
$$

with an initial condition

$$
u_{h}^{0}=u_{0}
$$


and boundary conditions

$$
u_{h}^{m+1}(a)=u_{h}^{m+1}(b)=0, \quad m=0,1, \ldots, M-1 .
$$

Now we prove the existence and uniqueness of solutions for (4.5) using the Brouwer fixed-point theorem.

Theorem 4.1. There exists a unique solution $u_{h}^{m+1} \in V_{h} \subset H_{0}^{\mu / 2}(\Omega)$ of (4.5)-(4.7).

Proof. Let

$$
G\left(u_{h}^{m+1}\right)=u_{h}^{m+1}-k \kappa_{\mu} \nabla^{\mu} u_{h}^{m+1}-k f\left(u_{h}^{m+1}\right)-u_{h}^{m}
$$

Then $G(v)$ is obviously a continuous function from $V_{h}$ to $V_{h}$. In order to show the existence of solution for $G(v)=0$, we adopt the mathematical induction. Assume that $u_{h}^{0}, u_{h}^{1}, \ldots, u_{h}^{m}$ exist for $m<M$. It follows from (1.8), Lemma 2.4, and Young's inequality that

$$
\begin{aligned}
(G(v), v) & =(v, v)-\left(u_{h}^{m}, v\right)-k\left(\kappa_{\mu} \nabla^{\mu} v, v\right)-k(f(v), v) \\
& \geq\|v\|_{L^{2}(\Omega)}^{2}-\left\|u_{h}^{m}\right\|_{L^{2}(\Omega)}\|v\|_{L^{2}(\Omega)}+k \kappa_{\mu}\left|\cos \left(\pi \cdot \frac{\mu}{2}\right)\right||v|_{H_{0}^{\mu / 2}(\Omega)}^{2}-C_{f} k\|v\|_{L^{2}(\Omega)}^{2} \\
& \geq\|v\|_{L^{2}(\Omega)}^{2}-\left\|u_{h}^{m}\right\|_{L^{2}(\Omega)}\|v\|_{L^{2}(\Omega)}-C_{f} k\|v\|_{L^{2}(\Omega)}^{2} \\
& \geq\|v\|_{L^{2}(\Omega)}^{2}-\frac{1}{2}\left(\left\|u_{h}^{m}\right\|_{L^{2}(\Omega)}^{2}+\|v\|_{L^{2}(\Omega)}^{2}\right)-C_{f} k\|v\|_{L^{2}(\Omega)}^{2} \\
& =\left(\frac{1}{2}-C_{f} k\right)\|v\|_{L^{2}(\Omega)}^{2}-\frac{1}{2}\left\|u_{h}^{m}\right\|_{L^{2}(\Omega)}^{2} .
\end{aligned}
$$

If we take sufficiently small $k$ so that $k<1 / 2 C_{f}$ and $\|v\|_{L^{2}(\Omega)}>\left\|u_{h}^{m}\right\|_{L^{2}(\Omega)} /\left(1-2 C_{f} k\right)$, then the Brouwer's fixed-point theorem implies the existence of a solution.

For the proof of the uniqueness of solutions, we assume that $u$ and $v$ are two solutions of (4.5). Then we obtain

$$
(u-v, \psi)=k \kappa_{\mu}\left(\nabla^{\mu}(u-v), \psi\right)+k(f(u)-f(v), \psi), \quad \forall \psi \in V_{h} \subset H_{0}^{\mu / 2}(\Omega)
$$

Replacing $\psi=u-v$ in the above equation and applying Lemma 2.4, we obtain

$$
\begin{aligned}
\|u-v\|_{L^{2}(\Omega)}^{2} & \leq-k \kappa_{\mu}\left|\cos \left(\pi \cdot \frac{\mu}{2}\right)\right||u-v|_{H_{0}^{\mu / 2}(\Omega)}+k\|f(u)-f(v)\|_{L^{2}(\Omega)}\|u-v\|_{L^{2}(\Omega)} \\
& \leq k\|f(u)-f(v)\|_{L^{2}(\Omega)}\|u-v\|_{L^{2}(\Omega)} \\
& \leq k C_{l}\|u-v\|_{L^{2}(\Omega)}^{2} .
\end{aligned}
$$

This implies $u-v=0$ since $u(0)=v(0)$. 
The following theorem presents the unconditional stability for (4.4).

Theorem 4.2. The fully discrete scheme (4.4) is unconditionally stable. In fact, for any $m$

$$
\left\|u^{m+1}\right\|_{L^{2}(\Omega)} \leq C\left\|u_{0}\right\|_{L^{2}(\Omega)}
$$

Proof. It follows from (1.8), Lemma 2.4, and Young's inequality that by taking $v=u^{m+1}$ in (4.4), we obtain

$$
\begin{aligned}
0= & \left(u^{m+1}, u^{m+1}\right)-k\left(\kappa_{\mu} \nabla^{\mu} u^{m+1}, u^{m+1}\right)-k\left(f\left(u^{m+1}\right), u^{m+1}\right)-\left(u^{m}, u^{m+1}\right) \\
\geq & \left\|u^{m+1}\right\|_{L^{2}(\Omega)}^{2}+k \kappa_{\mu}\left|\cos \left(\pi \cdot \frac{\mu}{2}\right) \| u^{m+1}\right|_{H_{0}^{\mu / 2}(\Omega)}^{2} \\
& -C_{f} k\left\|u^{m+1}\right\|_{L^{2}(\Omega)}^{2}-\left\|u^{m}\right\|_{L^{2}(\Omega)}\left\|u^{m+1}\right\|_{L^{2}(\Omega)} \\
\geq & \frac{1}{2}\left\|u^{m+1}\right\|_{L^{2}(\Omega)}^{2}+k \kappa_{\mu}\left|\cos \left(\pi \cdot \frac{\mu}{2}\right) \| u^{m+1}\right|_{H_{0}^{\mu / 2}(\Omega)}^{2} \\
& -C_{f} k\left\|u^{m+1}\right\|_{L^{2}(\Omega)}^{2}-\frac{1}{2}\left\|u^{m}\right\|_{L^{2}(\Omega)}^{2} .
\end{aligned}
$$

Then

$$
\begin{aligned}
\frac{1}{2}\left\|u^{m+1}\right\|_{L^{2}(\Omega)}^{2} & \leq \frac{1}{2}\left\|u^{m+1}\right\|_{L^{2}(\Omega)}^{2}+k \kappa_{\mu}\left|\cos \left(\pi \cdot \frac{\mu}{2}\right) \| u^{m+1}\right|_{H_{0}^{\mu / 2}(\Omega)}^{2} \\
& \leq C_{f} k\left\|u^{m+1}\right\|_{L^{2}(\Omega)}^{2}+\frac{1}{2}\left\|u^{m}\right\|_{L^{2}(\Omega)}^{2} .
\end{aligned}
$$

Adding the above inequality from $m=0$ to $m$, we obtain

$$
\left(1-2 C_{f} k\right)\left\|u^{m+1}\right\|_{L^{2}(\Omega)}^{2} \leq\left\|u_{0}\right\|_{L^{2}(\Omega)}^{2}+2 C_{f} k \sum_{j=1}^{m}\left\|u^{j}\right\|_{L^{2}(\Omega)}^{2}
$$

Applying the discrete Gronwall's inequality with sufficiently small $k$ such that $k<1 / 2 C_{f}$, we obtain the desired result.

The following theorem is an error estimate for the fully discrete problem (4.4).

Theorem 4.3. Let $u$ be the exact solution of (1.2) and let $u^{m}$ be the solution of (4.4). Then there is a constant $C$ such that

$$
\left\|u\left(t_{m}\right)-u^{m}\right\|_{L^{2}(\Omega)} \leq C k .
$$


Proof. Let $e^{m}=u\left(t_{m}\right)-u^{m}$ be the error at $t_{m}$. It follows from (1.2) and (4.4) that for any $v \in$ $H_{0}^{\mu / 2}(\Omega)$

$$
\left(e^{m+1}, v\right)-k\left(\kappa_{\mu} \nabla^{\mu} e^{m+1}, v\right)=k\left(f\left(u\left(t_{m+1}\right)\right)-f\left(u^{m+1}\right), v\right)+\left(e^{m}, v\right)+\left(k r^{m+1}, v\right)
$$

where $r=O(k)$. Taking $v=e^{m+1}$,

$$
\begin{aligned}
\left\|e^{m+1}\right\|_{L^{2}(\Omega)}^{2} \leq & \left\|e^{m+1}\right\|_{L^{2}(\Omega)}^{2}+k \kappa_{\mu}\left|\cos \left(\pi \cdot \frac{\mu}{2}\right) \| e^{m+1}\right|_{H_{0}^{\mu / 2}(\Omega)}^{2} \\
\leq & k\left\|f\left(u\left(t_{m+1}\right)\right)-f\left(u^{m+1}\right)\right\|_{L^{2}(\Omega)}\left\|e^{m+1}\right\|_{L^{2}(\Omega)} \\
& +\left\|e^{m}\right\|_{L^{2}(\Omega)}\left\|e^{m+1}\right\|_{L^{2}(\Omega)}+\left\|k r^{m+1}\right\|_{L^{2}(\Omega)}\left\|e^{m+1}\right\|_{L^{2}(\Omega)} .
\end{aligned}
$$

Applying the locally Lipschitz continuity of $f$ and Young's inequality, we obtain

$$
\begin{aligned}
\left\|e^{m+1}\right\|_{L^{2}(\Omega)}^{2} \leq & k C_{l}\left\|e^{m+1}\right\|_{L^{2}(\Omega)}^{2}+\left\|e^{m}\right\|_{L^{2}(\Omega)}\left\|e^{m+1}\right\|_{L^{2}(\Omega)}+\left\|k r^{m+1}\right\|_{L^{2}(\Omega)}\left\|e^{m+1}\right\|_{L^{2}(\Omega)} \\
\leq & k C_{l}\left\|e^{m+1}\right\|_{L^{2}(\Omega)}^{2}+\varepsilon_{1}\left\|e^{m}\right\|_{L^{2}(\Omega)}^{2}+\frac{1}{4 \varepsilon_{1}}\left\|e^{m+1}\right\|_{L^{2}(\Omega)}^{2} \\
& +\varepsilon_{2}\left\|k r^{m+1}\right\|_{L^{2}(\Omega)}^{2}+\frac{1}{4 \varepsilon_{2}}\left\|e^{m+1}\right\|_{L^{2}(\Omega)}^{2} .
\end{aligned}
$$

That is,

$$
\left(1-\frac{1}{4 \varepsilon_{1}}-\frac{1}{4 \varepsilon_{2}}\right)\left\|e^{m+1}\right\|_{L^{2}(\Omega)}^{2} \leq k C_{l}\left\|e^{m+1}\right\|_{L^{2}(\Omega)}^{2}+\varepsilon_{1}\left\|e^{m}\right\|_{L^{2}(\Omega)}^{2}+\varepsilon_{2}\left\|k r^{m+1}\right\|_{L^{2}(\Omega)}^{2}
$$

Denoting $\varepsilon_{0}=1-1 / 4 \varepsilon_{1}-1 / 4 \varepsilon_{2}$ and adding the above equation from $m=0$ to $m$, we obtain

$$
\left(\varepsilon_{0}-k C_{l}\right)\left\|e^{m+1}\right\|_{L^{2}(\Omega)}^{2} \leq \varepsilon_{1}\left\|e^{0}\right\|_{L^{2}(\Omega)}^{2}+\left(k C_{l}+\varepsilon_{1}-\varepsilon_{0}\right) \sum_{i=1}^{m}\left\|e^{i}\right\|_{L^{2}(\Omega)}^{2}+\varepsilon_{2} \sum_{i=1}^{m+1}\left\|k r^{i}\right\|_{L^{2}(\Omega)}^{2}
$$

Applying the discrete Gronwall's inequality with sufficiently small $k$ such that $\left(\varepsilon_{0}-\varepsilon_{1}\right) / C_{l}<$ $k<\varepsilon_{0} / C_{l}$, we obtain the desired result since $\sum_{i=1}^{m+1}\left\|k r^{i}\right\|_{L^{2}(\Omega)} \leq C k$ and $\left\|e^{0}\right\|_{L^{2}(\Omega)}=\| u(0)-$ $u^{0} \|_{L^{2}(\Omega)}=0$.

As in the previous section, denote $\theta^{m+1}=u_{h}^{m+1}-\tilde{u}_{h}^{m+1}$ and $\rho^{m+1}=\tilde{u}_{h}^{m+1}-u\left(t_{m+1}\right)$. Here $\tilde{u}_{h}^{m+1}$ is the elliptic projection of $u\left(t_{m+1}\right)$ defined in (3.13). Then

$$
e_{h}^{m+1}=\theta^{m+1}+\rho^{m+1}
$$


Theorem 4.4. Let $u$ be the exact solution of (1.2)-(1.4) and let $\left\{u_{h}^{m}\right\}_{m=0}^{M}$ be the solution of (4.5)(4.7). Then when $\mu \neq 3 / 2$

$$
\left\|u\left(t_{m+1}\right)-u_{h}^{m+1}\right\|_{L^{2}(\Omega)} \leq C k+C h^{\mu}\left\|u\left(t_{m+1}\right)\right\|_{H^{\mu}(\Omega)}
$$

and when $\mu=3 / 2,0<\epsilon<1 / 2$,

$$
\left\|u\left(t_{m+1}\right)-u_{h}^{m+1}\right\|_{L^{2}(\Omega)} \leq C k+C h^{\mu-\epsilon}\left\|u\left(t_{m+1}\right)\right\|_{H^{\mu-\epsilon}(\Omega)} .
$$

Proof. Since we know the estimates on $\rho$ from Lemma 3.1, we have only to show boundedness of $\theta^{m+1}$. Using the property (3.13), we obtain for $v \in V_{h}$

$$
\begin{aligned}
\left(\theta^{m+1}, v\right)-k\left(\kappa_{\mu} \nabla^{\mu} \theta^{m+1}, v\right)= & k\left(f\left(u_{h}^{m+1}\right)-f\left(u\left(t_{m+1}\right)\right), v\right)+\left(u_{h}^{m}-u\left(t_{m}\right), v\right) \\
& -\left(k r^{m+1}, v\right)-\left(\rho^{m+1}, v\right),
\end{aligned}
$$

where $r=O(k)$.

Taking $v=\theta^{m+1}$ and applying Lemma 2.4, the locally Lipschitz continuity of $f$, Young's inequality, and the triangle inequality, we obtain

$$
\begin{aligned}
\left\|\theta^{m+1}\right\|_{L^{2}(\Omega)}^{2} \leq & \left\|\theta^{m+1}\right\|_{L^{2}(\Omega)}^{2}+k \kappa_{\mu}\left|\cos \left(\pi \cdot \frac{\mu}{2}\right) \| \theta^{m+1}\right|_{H_{0}^{\mu / 2}(\Omega)}^{2} \\
\leq & k\left\|f\left(u_{h}^{m+1}\right)-f\left(u\left(t_{m+1}\right)\right)\right\|_{L^{2}(\Omega)}\left\|\theta^{m+1}\right\|_{L^{2}(\Omega)}+\left\|e_{h}^{m}\right\|_{L^{2}(\Omega)}\left\|\theta^{m+1}\right\|_{L^{2}(\Omega)} \\
& +\left\|k r^{m+1}\right\|_{L^{2}(\Omega)}\left\|\theta^{m+1}\right\|_{L^{2}(\Omega)}+\left\|\rho^{m+1}\right\|_{L^{2}(\Omega)}\left\|\theta^{m+1}\right\|_{L^{2}(\Omega)} \\
\leq & k C_{l}\left\|e_{h}^{m+1}\right\|_{L^{2}(\Omega)}\left\|\theta^{m+1}\right\|_{L^{2}(\Omega)} \\
& +\left(\left\|\theta^{m}\right\|_{L^{2}(\Omega)}+\left\|\rho^{m}\right\|_{L^{2}(\Omega)}\right)\left\|\theta^{m+1}\right\|_{L^{2}(\Omega)} \\
& +\left\|k r^{m+1}\right\|_{L^{2}(\Omega)}\left\|\theta^{m+1}\right\|_{L^{2}(\Omega)}+\left\|\rho^{m+1}\right\|_{L^{2}(\Omega)}\left\|\theta^{m+1}\right\|_{L^{2}(\Omega)} \\
\leq & k C_{l}\left(1+\frac{1}{4 \varepsilon_{6}}\right)\left\|\theta^{m+1}\right\|_{L^{2}(\Omega)}^{2}+\left(\frac{1}{4 \varepsilon_{3}}+\frac{1}{4 \varepsilon_{4}}+\frac{1}{4 \varepsilon_{5}}+\frac{1}{4 \varepsilon_{6}}\right)\left\|\theta^{m+1}\right\|_{L^{2}(\Omega)}^{2} \\
& +\varepsilon_{3}\left\|\theta^{m}\right\|_{L^{2}(\Omega)}^{2}+\varepsilon_{4}\left\|k r^{m+1}\right\|_{L^{2}(\Omega)}^{2}+\varepsilon_{5}\left\|\rho^{m}\right\|_{L^{2}(\Omega)}^{2}+\left(1+k C_{l}\right) \varepsilon_{6} \|_{\rho^{m+1} \|_{L^{2}(\Omega)}^{2}} .
\end{aligned}
$$


This implies that

$$
\begin{aligned}
(1- & \left.\frac{1}{4 \varepsilon_{3}}-\frac{1}{4 \varepsilon_{4}}-\frac{1}{4 \varepsilon_{5}}-\frac{1}{4 \varepsilon_{6}}\right)\left\|\theta^{m+1}\right\|_{L^{2}(\Omega)}^{2} \\
\leq & k C_{l}\left(1+\frac{1}{4 \varepsilon_{6}}\right)\left\|\theta^{m+1}\right\|_{L^{2}(\Omega)}^{2}+\varepsilon_{3}\left\|\theta^{m}\right\|_{L^{2}(\Omega)}^{2}+\varepsilon_{4}\left\|k r^{m+1}\right\|_{L^{2}(\Omega)}^{2} \\
& +\varepsilon_{5}\left\|\rho^{m}\right\|_{L^{2}(\Omega)}^{2}+\left(1+k C_{l}\right) \varepsilon_{6}\left\|\rho^{m+1}\right\|_{L^{2}(\Omega)}^{2} .
\end{aligned}
$$

Denote $\varepsilon_{7}=1-1 / 4 \varepsilon_{3}-1 / 4 \varepsilon_{4}-1 / 4 \varepsilon_{5}-1 / 4 \varepsilon_{6}$ and $\varepsilon_{8}=1+1 / 4 \varepsilon_{6}$. Then adding the above inequality from $m=0$ to $m$, we obtain

$$
\begin{aligned}
\left(\varepsilon_{7}-k C_{l} \varepsilon_{8}\right)\left\|\theta^{m+1}\right\|_{L^{2}(\Omega)}^{2} \leq & \varepsilon_{3}\left\|\theta^{0}\right\|_{L^{2}(\Omega)}^{2}+\left(k C_{l} \varepsilon_{8}+\varepsilon_{3}-\varepsilon_{7}\right) \sum_{i=1}^{m}\left\|\theta^{i}\right\|_{L^{2}(\Omega)}^{2} \\
& +\varepsilon_{4} \sum_{i=1}^{m+1}\left\|k r^{i}\right\|_{L^{2}(\Omega)}^{2}+\varepsilon_{5} \sum_{i=0}^{m}\left\|\rho^{i}\right\|_{L^{2}(\Omega)}^{2} \\
& +\left(1+k C_{l}\right) \varepsilon_{6} \sum_{i=1}^{m+1}\left\|\rho^{i}\right\|_{L^{2}(\Omega)}^{2}
\end{aligned}
$$

Applying the discrete Gronwall's inequality with sufficiently small $k$ such that $\left(\varepsilon_{7}-\varepsilon_{3}\right) /$ $\varepsilon_{8} C_{l}<k<\varepsilon_{7} / C_{l} \varepsilon_{8}$,

$$
\left\|\theta^{m+1}\right\|_{L^{2}(\Omega)}^{2} \leq C_{1}\left\|\theta^{0}\right\|_{L^{2}(\Omega)}^{2}+C_{2} \sum_{i=1}^{m+1}\left\|k r^{i}\right\|_{L^{2}(\Omega)}^{2}+C_{3} \sum_{i=0}^{m+1}\left\|\rho^{i}\right\|_{L^{2}(\Omega)}^{2}
$$

Also, using Lemma 3.1 and the initial conditions (1.3) and (4.6), we obtain

$$
\begin{aligned}
\left\|\theta^{0}\right\|_{L^{2}(\Omega)} & \leq\left\|u_{h}^{0}-u(0)\right\|_{L^{2}(\Omega)}+\left\|\tilde{u}_{h}^{0}-u(0)\right\|_{L^{2}(\Omega)} \\
& \leq C h^{\tilde{r}}\left\|u_{0}\right\|_{H^{\gamma}(\Omega)} .
\end{aligned}
$$

Since $\sum_{i=1}^{m+1}\left\|k r^{i}\right\|_{L^{2}(\Omega)} \leq C k$, we get

$$
\left\|\theta^{m+1}\right\|_{L^{2}(\Omega)} \leq C k+C(u) h^{\tilde{r}}
$$

where $\tilde{\gamma}=\mu$ if $\mu \neq 3 / 2$ and $\tilde{\gamma}=\mu-\epsilon, 0<\epsilon<1 / 2$, if $\mu=3 / 2$. Thus we obtain the desired result. 
Table 1: $L^{2}$-error and order of convergence in $x$ when $\mu=1.6$.

\begin{tabular}{|c|c|c|}
\hline \multirow{2}{*}{$h$} & \multicolumn{2}{|c|}{$\left\|\mathbf{u}-\mathbf{u}_{h}\right\|_{L^{2}(\Omega)}$} \\
\hline & Error & Order \\
\hline $1 / 4$ & $8.37811 e-03$ & - \\
\hline $1 / 8$ & $2.73537 e-03$ & 1.615 \\
\hline $1 / 16$ & $8.75752 e-04$ & 1.643 \\
\hline $1 / 32$ & $2.83167 e-04$ & 1.629 \\
\hline
\end{tabular}

\section{Numerical Experiments}

In this section, we present numerical results for the Galerkin approximations which supports the theoretical analysis discussed in the previous section.

Let $S_{h}$ denote a uniform partition of $\Omega$ and let $V_{h}$ denote the space of continuous piecewise linear functions defined on $S_{h}$. In order to implement the Galerkin finite element approximation, we adapt finite element discretization on the spatial axis and the backward Euler finite difference scheme along the temporal axis. We associate shape functions of space $V_{h}$ with the standard basis of the functions on the uniform interval with length $h$.

Example 5.1. We first consider a space fractional linear diffusion equation:

$$
\begin{aligned}
\frac{\partial u(x, t)}{\partial t}= & \nabla^{\mu} u(x, t)+\frac{2 t}{t^{2}+1} u(x, t)-\left(t^{2}+1\right) \\
& \times\left(\frac{\left\{x^{2-\mu}+(1-x)^{2-\mu}\right\}}{\Gamma(3-\mu)}-\frac{6\left\{x^{3-\mu}+(1-x)^{3-\mu}\right\}}{\Gamma(4-\mu)}+\frac{12\left\{x^{4-\mu}+(1-x)^{4-\mu}\right\}}{\Gamma(5-\mu)}\right)
\end{aligned}
$$

with an initial condition

$$
u(x, 0)=x^{2}(1-x)^{2}, \quad x \in[0,1]
$$

and boundary conditions

$$
u(0, t)=u(1, t)=0 .
$$

In this case, the exact solution is

$$
u(x, t)=\left(t^{2}+1\right) x^{2}(1-x)^{2} .
$$

Tables 1, 2, and 4 show the order of convergence and $L^{2}$-error between the exact solution and the Galerkin approximate solution of the fully discrete backward Euler method for (5.1) when $\mu=1.6, \mu=1.8$ and $\mu=1.5$, respectively. For numerical computation, the temporal step size $k=0.001$ is used in all three cases. Table 3 shows $L^{2}$-errors and orders of convergence for the Galerkin approximate solution when $\mu=1.8$ and the spatial step size $h=0.0625$. 
Table 2: $L^{2}$-error and order of convergence in $x$ when $\mu=1.8$.

\begin{tabular}{|c|c|c|}
\hline \multirow{2}{*}{$h$} & \multicolumn{2}{|c|}{$\left\|\mathbf{u}-\mathbf{u}_{h}\right\|_{L^{2}(\Omega)}$} \\
\hline & Error & Order \\
\hline $1 / 4$ & $8.03045 e-03$ & - \\
\hline $1 / 8$ & $2.28959 e-03$ & 1.810 \\
\hline $1 / 16$ & $6.32962 e-04$ & 1.855 \\
\hline $1 / 32$ & $1.76406 e-04$ & 1.843 \\
\hline
\end{tabular}

Table 3: $L^{2}$-error and order of convergence in $t$ when $\mu=1.8$.

\begin{tabular}{lccc}
\hline$k$ & Error & $\left\|\mathbf{u}-\mathbf{u}_{h}\right\|_{L^{2}(\Omega)}$ & Ratio \\
\hline $1 / 20$ & $4.20420 e-03$ & - \\
$1 / 30$ & $2.94873 e-03$ & 0.951 \\
$1 / 40$ & $2.31793 e-03$ & 0.954 \\
$1 / 50$ & $1.93046 e-03$ & 0.961 \\
\hline
\end{tabular}

Table 4: $L^{2}$-error and order of convergence in $x$ when $\mu=1.5$.

\begin{tabular}{lccc}
\hline$h$ & & $\left\|\mathbf{u}-\mathbf{u}_{h}\right\|_{L^{2}(\Omega)}$ & Order \\
\hline $1 / 4$ & Error & - \\
$1 / 8$ & $5.47750 e-03$ & 1.315 \\
$1 / 16$ & $2.20129 e-03$ & 1.312 \\
$1 / 32$ & $8.86858 e-04$ & 1.310 \\
\hline
\end{tabular}

According to Tables 1-3, we may find the order of convergence of $O\left(k+h^{\mu}\right)$ for this linear fractional diffusion problem (5.1)-(5.3) when $\mu \neq 3 / 2$. Furthermore, Table 4 shows orders of numerical convergence for the problem when $\mu=3 / 2$, where we may see that the order of convergence is of $O\left(k+h^{\mu-\epsilon}\right), 0<\epsilon<1 / 2$. It follows from Tables $1-4$ that numerical computations confirm the theoretical results.

We plot the exact solution and approximate solutions obtained by the backward Euler Galerkin method using $h=1 / 32$ and $k=1 / 1000$ for (5.1) with $\mu=1.6$ and $\mu=1.8$. Figure 1 shows the contour plots of an exact solution and numerical solutions at $t=1$, and Figure 2 shows log-log graph for the order of convergence.

Example 5.2. We consider a space fractional diffusion equation with a nonlinear Fisher type source term which is described as

$$
\frac{\partial u(x, t)}{\partial t}=\kappa_{\mu} \nabla^{\mu} u(x, t)+\lambda u(x, t)(1-\beta u(x, t))
$$




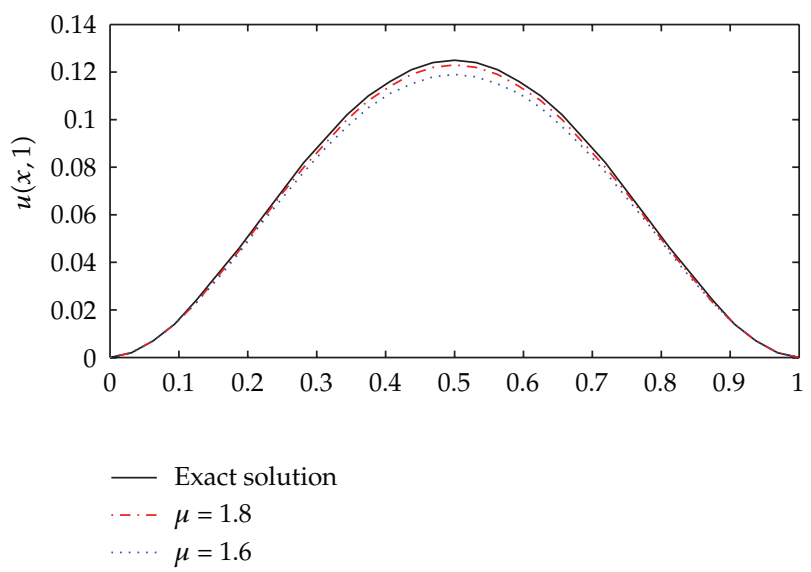

Figure 1: Exact and numerical solutions with $\mu=1.6$ and $\mu=1.8$.

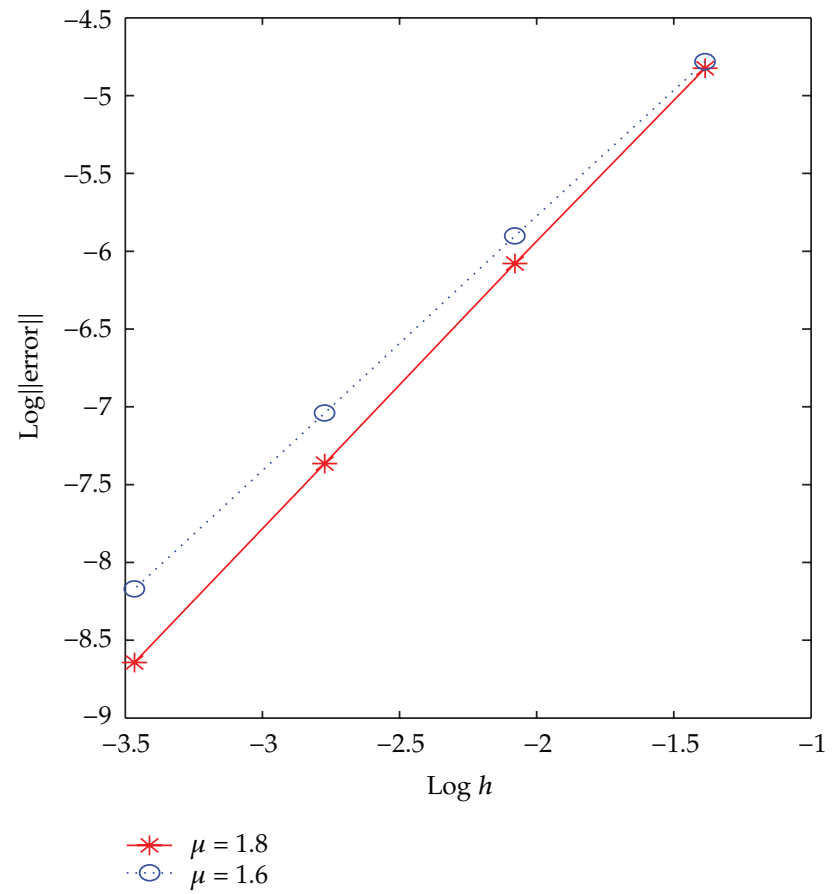

Figure 2: Log-log plots of the error for the rate of convergence.

with an initial condition

$$
u(x, 0)=u_{0}(x)
$$

and boundary conditions

$$
u(-1, t)=u(1, t)=0 .
$$




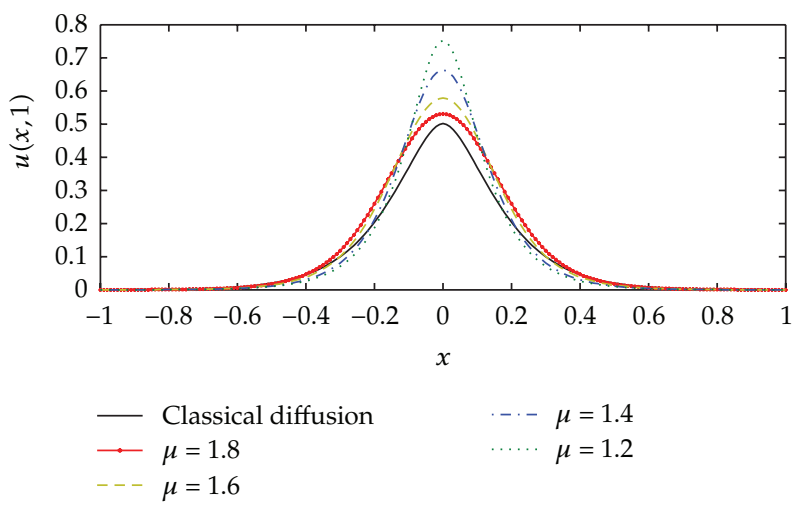

Figure 3: Numerical solutions for (5.5) with (5.8).

In fact, we will consider the case of $\kappa_{\mu}=0.1, \beta=1$ in (5.5) with an initial condition

$$
u_{0}(x)= \begin{cases}e^{-10 x}, & x \geq 0 \\ e^{10 x}, & x<0\end{cases}
$$

For numerical computations, we have to take care of the nonlinear term $f(u)=\ell u(1-$ $\beta u)$. This gives a complicated nonlinear matrix. In order to avoid the difficulty of solving nonlinear system, we adopted a linearized method replacing $\lambda u^{n+1}\left(1-\beta u^{n+1}\right)$ by $\lambda u^{n+1}\left(1-\beta u^{n}\right)$. Figure 3 shows contour plots of numerical solutions at $t=1$ for (5.5)-(5.8) with $\lambda=0.25$. For numerical computations, step sizes $h=0.01$ and $k=0.005$ are used. From the numerical results we may find that numerical solutions converge to the solution of classical diffusion equation as $\mu$ approaches to 2 .

Example 5.3. We now consider (5.5) with $\kappa_{\mu}=0.1, \beta=1$ and boundary conditions

$$
\lim _{|x| \rightarrow \infty} u(x, t)=0 .
$$

We will consider an initial condition with a sharp peak in the middle as

$$
u_{0}(x)=\operatorname{sech}^{2}(10 x)
$$

and an initial condition with a flat roof in the middle as

$$
u_{0}(x)= \begin{cases}e^{-10(x-1)}, & x>1 \\ 1, & -1<x \leq 1 \\ e^{10(x+1)}, & x \leq-1\end{cases}
$$

Tang and Weber [30] have obtained computational solutions for (5.5) with initial conditions (5.10) and (5.11) using a Petrov-Galerkin method when (5.5) is a classical diffusion 


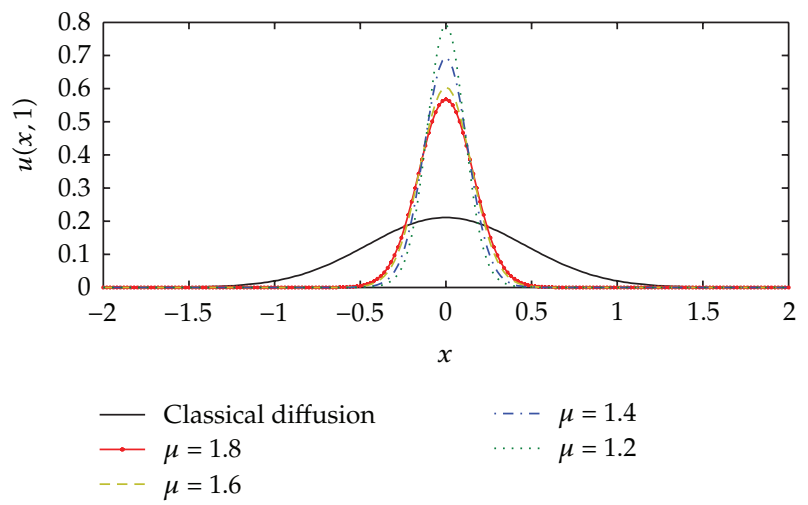

Figure 4: Numerical solutions at $t=1$ for (5.5) and (5.10) with $\lambda=0.25$.

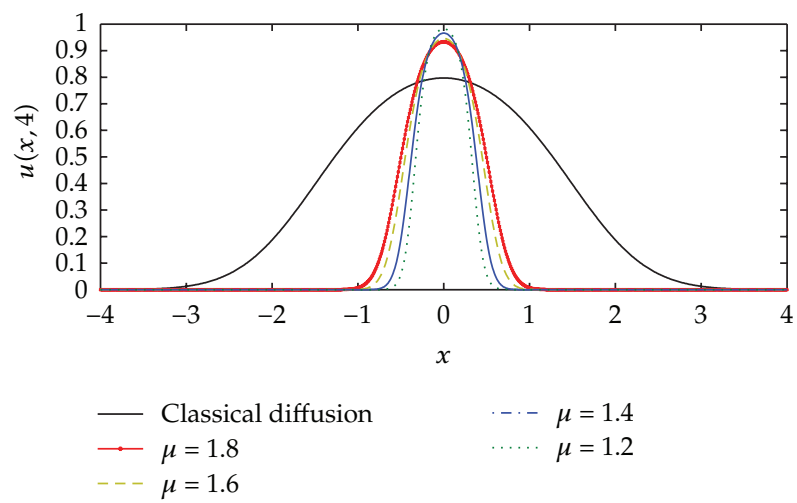

Figure 5: Numerical solutions at $t=4$ for (5.5) and (5.10) with $\lambda=1$.

problem. We obtain computational results using the method as in Example 5.2. Figure 4 shows contour plots of numerical solutions at $t=1$ for (5.5) with an initial condition (5.10) when $\Omega=(-2,2)$ and $\lambda=0.25$. Figure 5 shows also contour plots of numerical solutions at $t=4$ for (5.5) and (5.10) when $\Omega=(-4,4)$ and $\lambda=1$. In both cases, step sizes $h=0.01$ and $k=0.005$ are used for computation. According to Figures 4 and 5, we may see that the diffusivity depends on $\mu$ but it is far less than that of the classical solution. That is, the fractional diffusion problem keeps the peak in the middle for longer time than the classical one does.

Figure 6 shows contour plots of numerical solutions for (5.5) with an initial condition (5.10) when $\mu=1.8, \Omega=(-2,2)$ and $\lambda=1$. In this case, step sizes $h=0.01$ and $k=0.005$ are also used for computation. But the period of time is from $t=0$ to $t=5$. According to Figure 6, we may see that the peak goes down rapidly for a short time, and it begins to go up after the contour arrives at the lowest level.

Figure 7 shows contour plots of numerical solutions at $t=1$ for (5.5) with an initial condition (5.11) when $\Omega=(-4,4)$ and $\lambda=0.25$. In this case, step sizes $h=0.01$ and $k=$ 0.005 are also used for computation. According to Figure 7, we may find that the fractional diffusion problem keeps the flat roof in the middle for longer time than the classical one does. 


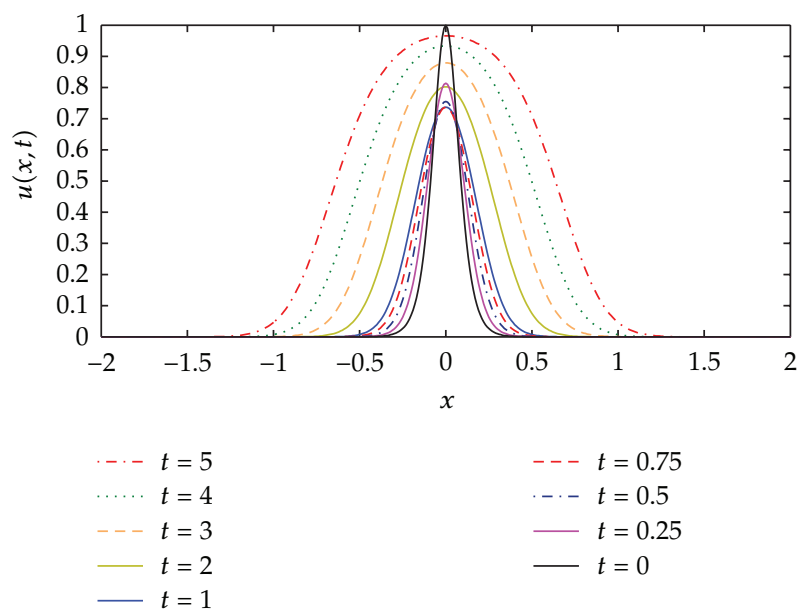

Figure 6: Numerical solutions for (5.5) and (5.10) with $\lambda=1$.

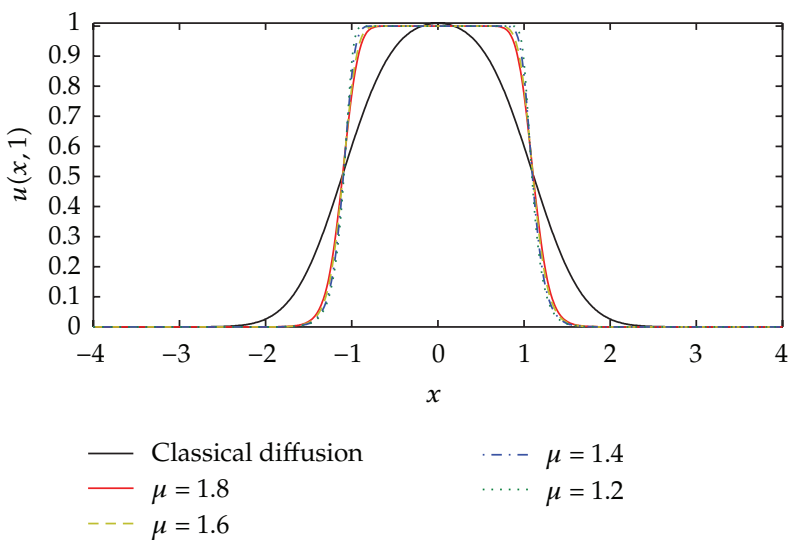

Figure 7: Numerical solutions for (5.5) and (5.11) with $\lambda=0.25$.

\section{Concluding Remarks}

Galerkin finite element methods are considered for the space fractional diffusion equation with a nonlinear source term. We have derived the variational formula of the semidiscrete scheme by using the Galerkin finite element method in space. We showed existence and stability of solutions for the semidiscrete scheme. Furthermore, we derived the fully time-space discrete variational formulation using the backward Euler method. Existence and uniqueness of solutions for the fully discrete Galerkin method have been discussed. Also we proved that the scheme is unconditionally stable, and it has the order of convergence of $O\left(k+h^{\tilde{r}}\right)$, where $\tilde{\gamma}$ is a constant depending on the order of fractional derivative. Numerical computations confirm the theoretical results discussed in the previous section for the problem with a linear source term. For the fractional diffusion problem with a nonlinear source term, we may find that the diffusivity depends on the order of fractional derivative, and numerical solutions of fractional order problems are less diffusive than the solution of a classical diffusion problem. 


\section{Acknowledgment}

The authors would like to express sincere thanks to the referee for their invaluable comments.

\section{References}

[1] K. Diethelm and N. J. Ford, "Analysis of fractional differential equations," Journal of Mathematical Analysis and Applications, vol. 265, no. 2, pp. 229-248, 2002.

[2] A. A. Kilbas and J. J. Trujillo, "Differential equations of fractional order: methods, results and problems. I," Applicable Analysis, vol. 78, no. 1-2, pp. 153-192, 2001.

[3] A. A. Kilbas and J. J. Trujillo, "Differential equations of fractional order: methods, results and problems. II," Applicable Analysis, vol. 81, no. 2, pp. 435-493, 2002.

[4] R. Metzler and J. Klafter, “The random walk's guide to anomalous diffusion: a fractional dynamics approach," Physics Reports, vol. 339, no. 1, pp. 1-77, 2000.

[5] R. Metzler and J. Klafter, "The restaurant at the end of the random walk: recent developments in the description of anomalous transport by fractional dynamics," Journal of Physics A, vol. 37, no. 31, pp. R161-R208, 2004.

[6] K. B. Oldham and J. Spanier, The Fractional Calculus, Dover Publications, New York, NY, USA, 2002.

[7] I. Podlubny, Fractional Differential Equations: An Introduction to Fractional Derivatives, Fractional Differential Equations, to Methods of Their Solution and Some of Their Applications, vol. 198 of Mathematics in Science and Engineering, Academic Press, San Diego, Calif, USA, 1999.

[8] B. Baeumer, M. Kovács, and M. M. Meerschaert, "Numerical solutions for fractional reaction-diffusion equations," Computers \& Mathematics with Applications, vol. 55, no. 10, pp. 2212-2226, 2008.

[9] W. Deng, "Finite element method for the space and time fractional Fokker-Planck equation," SIAM Journal on Numerical Analysis, vol. 47, no. 1, pp. 204-226, 2008.

[10] Z. Q. Deng, V. P. Singh, and L. Bengtsson, "Numerical solution of fractional advection-dispersion equation," Journal of Hydraulic Engineering, vol. 130, no. 5, pp. 422-431, 2004.

[11] Y. Lin and C. Xu, "Finite difference/spectral approximations for the time-fractional diffusion equation," Journal of Computational Physics, vol. 225, no. 2, pp. 1533-1552, 2007.

[12] F. Liu, A. Anh, and I. Turner, "Numerical solution of the space fractional Fokker-Planck equation," Journal of Computational and Applied Mathematics, vol. 166, pp. 209-219, 2004.

[13] B. Baeumer, M. Kovács, and M. M. Meerschaert, "Fractional reproduction-dispersal equations and heavy tail dispersal kernels," Bulletin of Mathematical Biology, vol. 69, no. 7, pp. 2281-2297, 2007.

[14] M. M. Meerschaert and C. Tadjeran, "Finite difference approximations for fractional advection-dispersion flow equations," Journal of Computational and Applied Mathematics, vol. 172, no. 1, pp. 65-77, 2004.

[15] M. M. Meerschaert, H.-P. Scheffler, and C. Tadjeran, "Finite difference methods for two-dimensional fractional dispersion equation," Journal of Computational Physics, vol. 211, no. 1, pp. 249-261, 2006.

[16] M. M. Meerschaert and C. Tadjeran, "Finite difference approximations for two-sided space-fractional partial differential equations," Applied Numerical Mathematics, vol. 56, no. 1, pp. 80-90, 2006.

[17] V. E. Lynch, B. A. Carreras, D. del-Castillo-Negrete, K. M. Ferreira-Mejias, and H. R. Hicks, "Numerical methods for the solution of partial differential equations of fractional order," Journal of Computational Physics, vol. 192, no. 2, pp. 406-421, 2003.

[18] H. W. Choi, S. K. Chung, and Y. J. Lee, “Numerical solutions for space fractional dispersion equations with nonlinear source terms," Bulletin of the Korean Mathematical Society, vol. 47, no. 6, pp. 1225-1234, 2010.

[19] W. Deng, "Numerical algorithm for the time fractional Fokker-Planck equation," Journal of Computational Physics, vol. 227, no. 2, pp. 1510-1522, 2007.

[20] W. Deng and C. Li, "Finite difference methods and their physical constraints for the fractional KleinKramers equation," Numerical Methods for Partial Differential Equations, vol. 27, no. 6, pp. 1561-1583, 2011.

[21] T. A. M. Langlands and B. I. Henry, "The accuracy and stability of an implicit solution method for the fractional diffusion equation," Journal of Computational Physics, vol. 205, no. 2, pp. 719-736, 2005.

[22] F. Liu, P. Zhuang, V. Anh, I. Turner, and K. Burrage, "Stability and convergence of the difference methods for the space-time fractional advection-diffusion equation," Applied Mathematics and Computation, vol. 191, no. 1, pp. 12-20, 2007. 
[23] P. Zhuang, F. Liu, V. Anh, and I. Turner, "New solution and analytical techniques of the implicit numerical method for the anomalous subdiffusion equation," SIAM Journal on Numerical Analysis, vol. 46, no. 2, pp. 1079-1095, 2008.

[24] V. J. Ervin and J. P. Roop, "Variational formulation for the stationary fractional advection dispersion equation," Numerical Methods for Partial Differential Equations, vol. 22, no. 3, pp. 558-576, 2006.

[25] J. P. Roop, "Computational aspects of FEM approximation of fractional advection dispersion equations on bounded domains in $\mathbf{R}^{2}$," Journal of Computational and Applied Mathematics, vol. 193, no. 1, pp. 243-268, 2006.

[26] V. J. Ervin, N. Heuer, and J. P. Roop, "Numerical approximation of a time dependent, nonlinear, spacefractional diffusion equation," SIAM Journal on Numerical Analysis, vol. 45, no. 2, pp. 572-591, 2007.

[27] D. Braess, Finite Elements, Cambridge University Press, Cambridge, UK, 2nd edition, 2001.

[28] S. C. Brenner and L. R. Scott, The Mathematical Theory of Finite Element Methods, vol. 15 of Texts in Applied Mathematics, Springer, New York, NY, USA, 1994.

[29] Z. Bai and H. Lü, "Positive solutions for boundary value problem of nonlinear fractional differential equation," Journal of Mathematical Analysis and Applications, vol. 311, no. 2, pp. 495-505, 2005.

[30] S. Tang and R. O. Weber, "Numerical study of Fisher's equation by a Petrov-Galerkin finite element method," Australian Mathematical Society B, vol. 33, no. 1, pp. 27-38, 1991. 


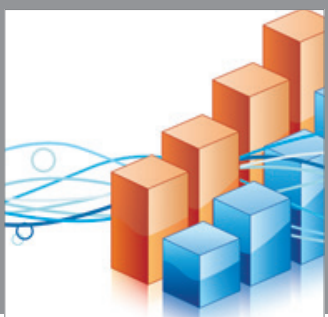

Advances in

Operations Research

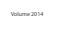

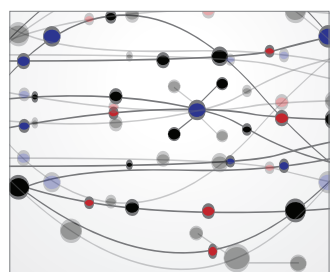

\section{The Scientific} World Journal
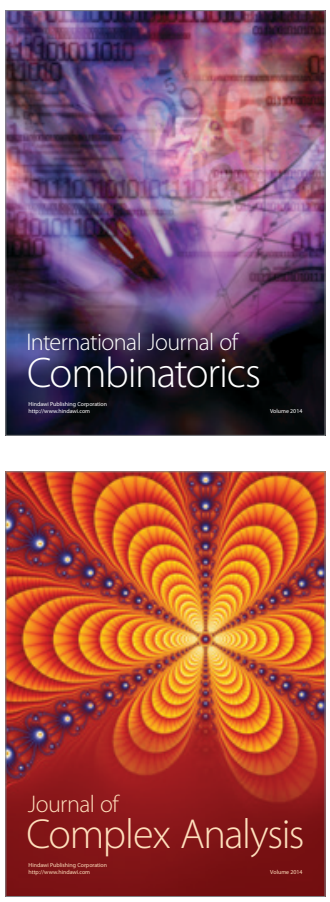

International Journal of

Mathematics and

Mathematical

Sciences
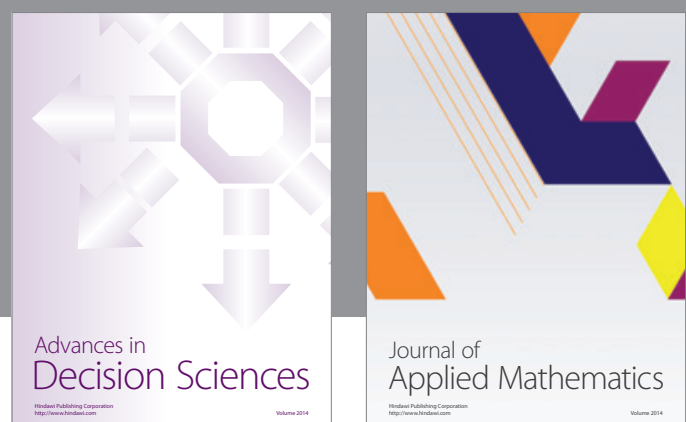

Journal of

Applied Mathematics
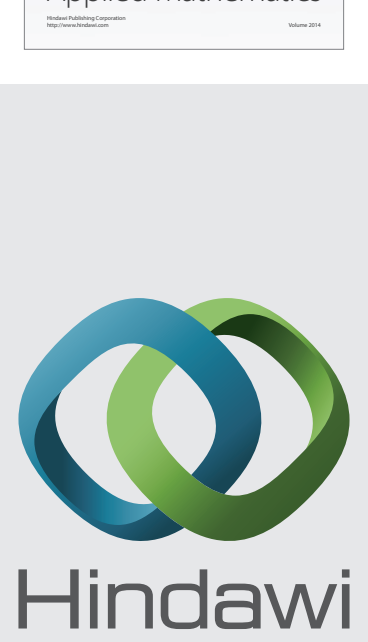

Submit your manuscripts at http://www.hindawi.com
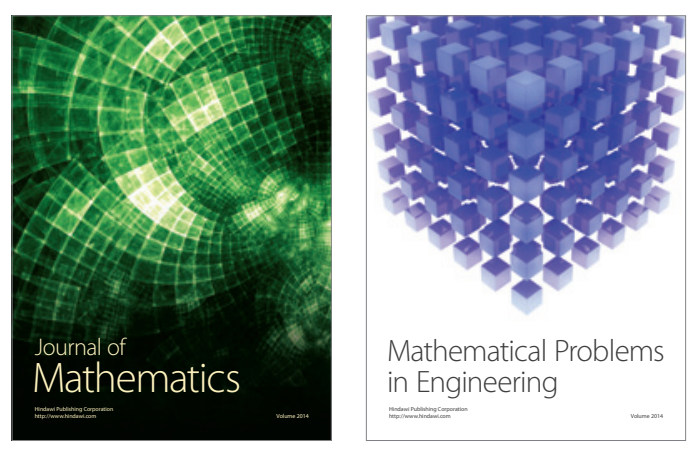

Mathematical Problems in Engineering
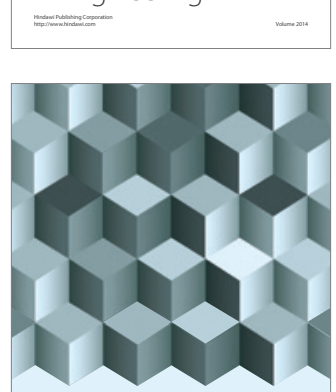

Journal of

Function Spaces
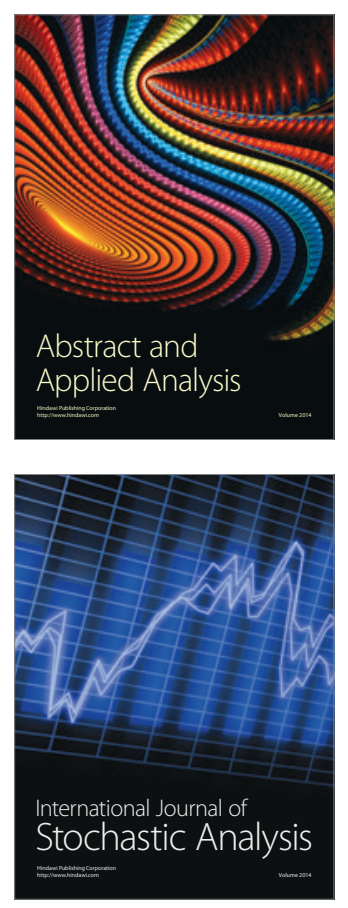

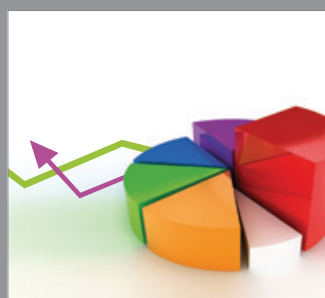

ournal of

Probability and Statistics

Promensencen
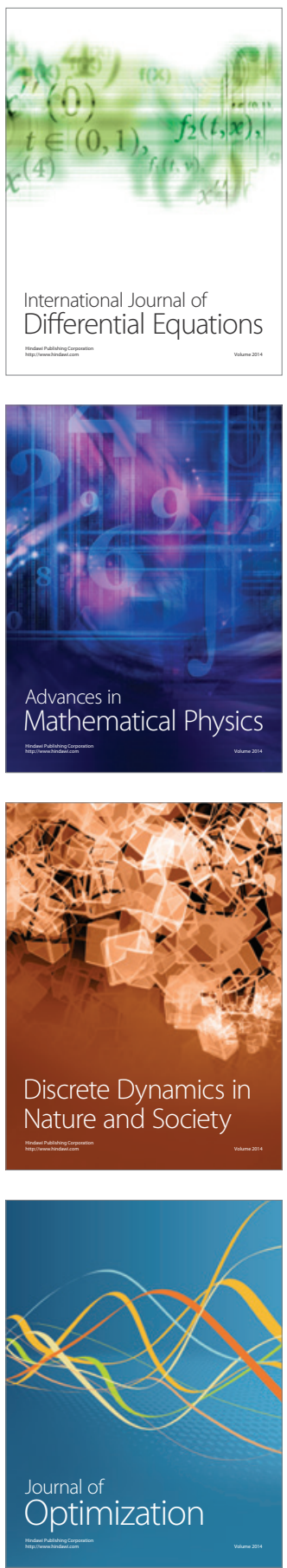